PROCEEDINGS OF THE

AMERICAN MATHEMATICAL SOCIETY

Volume 129, Number 7 , Pages 2103-2109

S 0002-9939(00)05728-2

Article electronically published on November 21, 2000

\title{
PROJECTIONS OF POLYTOPES ON THE PLANE AND THE GENERALIZED BAUES PROBLEM
}

\author{
CHRISTOS A. ATHANASIADIS
}

(Communicated by John R. Stembridge)

\begin{abstract}
Given an affine projection $\pi: P \rightarrow Q$ of a $d$-polytope $P$ onto a polygon $Q$, it is proved that the poset of proper polytopal subdivisions of $Q$ which are induced by $\pi$ has the homotopy type of a sphere of dimension $d-3$ if $\pi$ maps all vertices of $P$ into the boundary of $Q$. This result, originally conjectured by Reiner, is an analogue of a result of Billera, Kapranov and Sturmfels on cellular strings on polytopes and explains the significance of the interior point of $Q$ present in the counterexample to their generalized Baues conjecture, constructed by Rambau and Ziegler.
\end{abstract}

\section{INTRODUCTION}

Motivated by their theory of fiber polytopes [6] and [18, Lecture 9], Billera and Sturmfels have associated to any affine projection of convex polytopes $\pi: P \rightarrow Q$ the Baues poset $\omega(P \stackrel{\pi}{\rightarrow} Q)$ of proper polytopal subdivisions of $Q$ which are induced by $\pi$. This poset reduces to the poset of proper cellular strings [7] on $P$ with respect to $\pi$, if $\operatorname{dim}(Q)=1$, and can be described in general as the poset of proper "locally $\pi$-coherent cellular strings" on $P$ [7, 13, which is a combinatorial model for the space of all continuous sections of $\pi$ lying in the boundary of $P$. It provides a common framework in which one can study traditional objects in polyhedral combinatorics, such as monotone paths, triangulations of point configurations and zonotopal tilings, and their connectivity by polygon moves, geometric bistellar operations and mutations, respectively. For the relevance of polytopal subdivisions in various areas of mathematics, see [12, Chapter 1].

The connectivity of the Baues poset, in a topological sense, is the subject of the generalized Baues problem, posed by Billera, Kapranov and Sturmfels [7] (see 14] for a survey). Specifically, the problem asks to determine whether the poset $\omega(P \stackrel{\pi}{\rightarrow} Q)$, endowed with a standard topology [8], has the homotopy type of a sphere of $\operatorname{dimension} \operatorname{dim}(P)-\operatorname{dim}(Q)-1$. An affirmative answer was given in [7] when $\operatorname{dim}(Q)=1$, proving in particular a conjecture of Baues [5] in the theory of loop spaces. However, an explicit example of a projection $\pi$ for which $\omega(P \stackrel{\pi}{\rightarrow} Q)$ is disconnected was constructed by Rambau and Ziegler [13] and the borderline of positive and negative answers remains an intriguing area of research (see [16] for a

Received by the editors September 29, 1999 and, in revised form, October 22, 1999.

2000 Mathematics Subject Classification. Primary 52B11; Secondary 06A07, 55P15.

The author's research was supported by the Göran Gustafsson Foundation at the Royal Institute of Technology, Stockholm, Sweden. 
more recent negative result). In the counterexample of [13], in which $P$ and $Q$ have dimensions five and two, respectively, the image of one of the vertices of $P$ under $\pi$ is an interior point of the polygon $Q$. Our main result explains the significance of this fact.

Theorem 1.1. Let $\pi: P \rightarrow Q$ be an affine projection of a d-polytope $P$ onto a polygon $Q$. If $\pi$ maps all vertices of $P$ into the boundary of $Q$, then the Baues poset $\omega(P \stackrel{\pi}{\rightarrow} Q)$ has the homotopy type of a $(d-3)$-sphere.

Theorem [1.1, which can be viewed as an analogue of the main result of [7], was conjectured by Reiner [14, Conjecture 6.6]. In fact Reiner conjectures a stronger statement, namely that the subposet of $\pi$-coherent proper subdivisions is a strong deformation retract of $\omega(P \stackrel{\pi}{\rightarrow} Q)$ under the same assumptions on $\pi$. This subposet of coherent subdivisions is isomorphic to the poset of proper faces of the fiber polytope [6] associated to $\pi$, a polytope of dimension $d-2$, and is hence homeomorphic to a $(d-3)$-sphere. In the case $\operatorname{dim}(Q)=2$, the generalized Baues problem is also known to have an affirmative answer when $P$ has dimension $d \leq 4$ [13], when $P$ is a cube [17, when $P$ is a simplex 10 and $\pi$ maps the vertices of $P$ to points in general position in $\mathbb{R}^{2}$ and when $P$ has $d+2$ vertices and $\pi$ satisfies a condition weaker than the one in Theorem 1.1. (see [1, Theorem 1.2]).

We prove Theorem 1.1 in Section 3 after we introduce the necessary definitions and background in Section 2. Our method is an analogue in two dimensions of the method used in [2] to give a new proof and generalization to the main result of [7]. We also derive a connectivity result on triangulations of $Q$ by diagonal flips as a corollary, following reasoning from [14 Section 3].

\section{BACKGROUND}

In this section we provide the necessary definitions and background. Any undefined terminology relating to convex polytopes can be found in Ziegler's text [18.

2.1. The Baues poset. Let $\pi: P \rightarrow Q$ be an affine projection of a polytope $P$ onto a polygon $Q \subseteq \mathbb{R}^{2}$ and let $\mathcal{A}$ be the point configuration of projections of the vertices of $P$ in $\mathbb{R}^{2}$. Thus $\mathcal{A}$ is a multiset of points, each labeled with the corresponding vertex of $P$, and $\operatorname{conv}(\mathcal{A})=Q$.

A face of a subconfiguration $\sigma \subseteq \mathcal{A}$ is a subconfiguration of $\sigma$ consisting of all points on which some linear functional in $\mathbb{R}^{2}$ takes its minimum over $\sigma$. We call $\sigma$ spanning if $\operatorname{conv}(\sigma)$ has dimension two. A proper (polytopal) subdivision of $\mathcal{A}$ is a finite collection $x$ of spanning, proper subconfigurations of $\mathcal{A}$, called the cells of $x$, such that:

(i) $\bigcup_{\sigma \in x} \operatorname{conv}(\sigma)=Q$

and for any two $\sigma_{1}, \sigma_{2} \in x$,

(ii) $\operatorname{conv}\left(\sigma_{1}\right) \cap \operatorname{conv}\left(\sigma_{2}\right)=\operatorname{conv}\left(\sigma_{1} \cap \sigma_{2}\right)$ and

(iii) $\sigma_{1} \cap \sigma_{2}$ is either empty or a face of both $\sigma_{1}$ and $\sigma_{2}$.

A subconfiguration $\sigma \subseteq \mathcal{A}$ is $\pi$-induced if the corresponding set of vertices of $P$ spans a face of $P$ and a subdivision $x$ of $\mathcal{A}$ is $\pi$-induced if so is every cell of $x$. The Baues poset $\omega(P \stackrel{\pi}{\rightarrow} Q)[6$, 7] is the set of proper $\pi$-induced subdivisions of $\mathcal{A}$, ordered by refinement, i.e. by letting $x \leq y$ if for every $\sigma \in x$ there exists a $\tau \in y$ with $\sigma \subseteq \tau$. If $P$ is a simplex, then this is the refinement poset of all proper 
polytopal subdivisions of $\mathcal{A}$. If $\mathcal{B} \subseteq \mathcal{A}$ is a spanning subconfiguration, we denote by $\omega_{\pi}(\mathcal{B})$ the refinement poset of all $\pi$-induced subdivisions of $\mathcal{B}$, i.e. subdivisions $x$ of $\mathcal{B}$ such that each cell of $x$ is $\pi$-induced. By definition this poset contains the trivial subdivision, which consists only of the cell $\mathcal{B}$, if $\mathcal{B}$ is $\pi$-induced.

The previous definitions extend naturally when $Q$ has any dimension. For a definition of induced subdivisions as collections of faces of $P$, see [18, Section 9.1], [13] or [1, Section 2].

2.2. Poset topology. When referring to the topology of a finite poset $\omega$ we mean the topology of the geometric realization of its order complex $\Delta(\omega)$, i.e. the simplicial complex of chains in $\omega\left[\underline{8}\right.$. For $x \in \omega$ we write $\omega_{\leq x}=\{y \in \omega: y \leq x\}$. The following variation of the Quillen Fiber Lemma [1] will be useful in relating homotopy types of posets.

Lemma 2.1 (Babson [4]). Let $f: \omega \rightarrow \omega^{\prime}$ be an order preserving map of posets. If

(i) $f^{-1}(y)$ is contractible for every $y \in \omega^{\prime}$ and

(ii) $\omega_{\leq x} \cap f^{-1}(y)$ is contractible for every $x \in \omega$ and $y \in \omega^{\prime}$ with $f(x)>y$,

then $f$ induces a homotopy equivalence.

We say that a poset $\omega$ is connected if its Hasse diagram is connected as a graph.

2.3. Face and flag cuts. Given a proper face $F$ of a $d$-polytope $P \subseteq \mathbb{R}^{d}$, let $H$ be a supporting hyperplane of $F$, given by the equation $c \cdot x=c_{0}$, so that $c \cdot x \geq c_{0}$ for $x \in P$. We call the intersection of $P$ with the hyperplane $c \cdot x=c_{0}+\delta$ for some small $\delta>0$ the face cut of $P$ at $F$. This is a polytope which reduces to the vertex figure [18, p. 54] of $P$ at $v$ if $F$ is a vertex $v$ of $P$. Its face poset is the set of faces of $P$ which intersect both $F$ and $P-F$, ordered by inclusion, and hence its combinatorial type is independent of $\delta$.

By iterating this construction we get the following lemma.

Lemma 2.2. Let $\emptyset=F_{0} \subset F_{1} \subset \cdots \subset F_{k-1} \subset F_{k}=P$ be a flag $\mathcal{F}$ of faces of the $d$-polytope $P$. The set of faces of $P$ which intersect $F_{i}-F_{i-1}$ for all $1 \leq i \leq k$, ordered by inclusion, is the face poset of a polytope of dimension $d-k+1$.

We call this polytope the flag cut of $P$ at $\mathcal{F}$. Note that the flag cut reduces to the face figure [18 Problem 2.9] of $P$ at $F_{k-1}$ if $\operatorname{dim}\left(F_{i}\right)=i-1$ for each $1 \leq i<k$.

The following elementary lemma will be needed in Section 3 ,

Lemma 2.3. If $P \subseteq \mathbb{R}^{d}$ is a polytope and $U$ is an open halfspace in $\mathbb{R}^{d}$ which intersects $P$, then the poset $\omega$ of faces of $P$ which lie in $U$, ordered by inclusion, is contractible.

Proof. Let $u$ be one of the vertices of $P$ which lies in $U$ and either minimizes or maximizes the linear functional associated to $U$. Let $\mathcal{F}$ be the polytopal complex of faces of $P$ contained in $U$ and note that $\Delta(\omega)$ is the barycentric subdivision of $\mathcal{F}$. If $P \subseteq U$, then $\omega$ has a maximum element and hence is contractible. Otherwise, there is a stereographic projection $h$ which maps homeomorphically the union of the faces of $\mathcal{F}$ onto a closed subset of the boundary of $U$ which is star convex with respect to the point $h(u)$. Hence this union is contractible and thus, so is $\omega$. 


\section{HoMOTOPY TYPE AND CONNECTIVITY}

Throughout this section, $P \subseteq \mathbb{R}^{d}$ and $Q \subseteq \mathbb{R}^{2}$ are full dimensional polytopes, $\pi: P \rightarrow Q$ is as in Theorem 1.1 and $\mathcal{A}$ is the point configuration in $\mathbb{R}^{2}$ of projected vertices of $P$, as in Section 2.1, each lying in the boundary of $Q$.

Let $\tau \subseteq \mathcal{A}$ be $\pi$-induced and such that $\operatorname{conv}(\tau)$ is a line segment. If $\mathcal{B}$ is spanning with $\tau \subseteq \mathcal{B} \subseteq \mathcal{A}$, we denote by $\mathrm{lk}_{\pi}(\tau, \mathcal{B})$ the set of spanning, $\pi$-induced $\sigma \subseteq \mathcal{B}$ which have $\tau$ as a face and partially order this set by inclusion.

Lemma 3.1. Let $\tau \subseteq \mathcal{A}$ be $\pi$-induced, as above, and let $e$ be the line segment $\operatorname{conv}(\tau)$. Suppose that $\mathcal{B}$ is either

(i) $\mathcal{A}$ itself, if $e$ is contained in the boundary of $Q$ or

(ii) the configuration of points in $\mathcal{A}$ which lie in one of the two polygons into which $Q$ is divided by e, if e intersects the interior of $Q$,

so that $\tau \subseteq \mathcal{B} \subseteq \mathcal{A}$ and e lies in the boundary of $\operatorname{conv}(\mathcal{B})$. Then the poset $\operatorname{lk}_{\pi}(\tau, \mathcal{B})$ is nonempty and contractible.

Proof. Let $L$ and $L^{+}$be the line defined by $e$ and the closed halfplane defined by $L$ which contains $\mathcal{B}$, respectively. The set $\pi^{-1}\left(L^{+}\right)$is a closed halfspace $H^{+}$in $\mathbb{R}^{d}$ defined by the hyperplane $H=\pi^{-1}(L)$. Let $F$ be the face of $P$ which induces $\tau$, so that $F \subseteq H$.

By definition, $\mathrm{lk}_{\pi}(\tau, \mathcal{B})$ is isomorphic to the poset of faces $G$ of $P$ such that $F \subset G \subseteq H^{+}$and $H \cap G=F$, ordered by inclusion. Since the poset of faces of $P$ which contain $F$ is the face poset of the face figure $P_{F}$ of $P$ at $F$, it follows that $\mathrm{lk}_{\pi}(\tau, \mathcal{B})$ is isomorphic to the poset of faces of the polytope $P_{F}$ which lie in an open halfspace which intersects $P_{F}$. This poset is contractible by Lemma 2.3 .

For point configurations $\mathcal{B} \subseteq \mathcal{C}$, we say (with a slight abuse of language) that a subdivision of $\mathcal{B}$ refines one of $\mathcal{C}$ if every cell of the former subdivision is contained in a cell of the latter.

Lemma 3.2. Let $\tau$ and $\mathcal{B}$ be as in case (ii) of Lemma 3.1 and let $X$ be a subdivision in $\omega_{\pi}(\mathcal{C})$ (possibly trivial), for some $\mathcal{C}$ with $\mathcal{B} \subseteq \mathcal{C} \subseteq \mathcal{A}$, such that $\tau$ is contained in a cell of $X$.

Then the subposet $\omega_{X}(\tau, \mathcal{B})$ of $\omega_{\pi}(\mathcal{B})$, consisting of the subdivisions $x$ such that $x$ refines $X$ and $\tau$ is a face of a cell of $x$, is contractible.

Proof. We proceed by induction on the cardinality of $\mathcal{B}$. Let $\sigma$ be the unique cell of $X$ which contains $\tau$ and at least one point of $\mathcal{B}$ not in $\operatorname{conv}(\tau)$. Let $\omega=\omega_{X}(\tau, \mathcal{B})$, $\sigma^{\prime}=\sigma \cap \mathcal{B}$ and $\omega^{\prime}=\mathrm{lk}_{\pi}\left(\tau, \sigma^{\prime}\right)$. For $x \in \omega$, let $f(x)$ be the unique cell in $x$ which has $\tau$ as a face. Clearly $f(x) \in \omega^{\prime}$ and $f: \omega \rightarrow \omega^{\prime}$ is an order preserving map of posets. By Lemma 3.1 applied to the restriction of $\pi$ to the face of $P$ which induces $\sigma, \omega^{\prime}$ is nonempty and contractible. Hence it suffices to show that the two conditions of Lemma 2.1 are satisfied.

To verify condition (i), let $y \in \omega^{\prime}$ and $\tau(y)$ be the set of 1-faces of $y$ other than $\tau$ whose convex hull intersects the interior of $Q$ (see Figure 1a). For $\tau^{\prime} \in \tau(y)$, let $\mathcal{B}_{\tau^{\prime}} \subset \mathcal{B}$ be the point configuration assigned to $\tau^{\prime}$ in case (ii) of Lemma 3.1. Note that each $\tau^{\prime} \in \tau(y)$ is $\pi$-induced and contained in the cell $\sigma$ of $X$ (since so is $y$ ) and that any subdivision in $f^{-1}(y)$ must contain a cell other than $y$ having $\tau^{\prime}$ as a face. It follows that $f^{-1}(y)$ is the direct product of the posets $\omega_{X}\left(\tau^{\prime}, \mathcal{B}_{\tau^{\prime}}\right)$, to which the induction hypothesis applies, and hence is contractible. Similarly for condition (ii), if $x \in \omega$ satisfies $f(x)>y$, then each $\tau^{\prime} \in \tau(y)$ is contained in the cell $f(x)$ of $x$ 


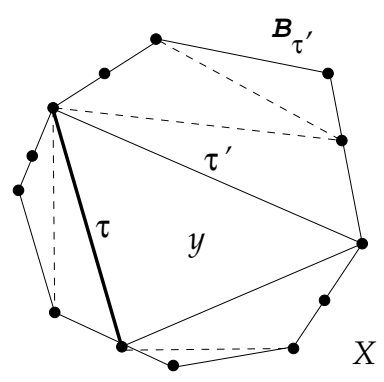

(a)

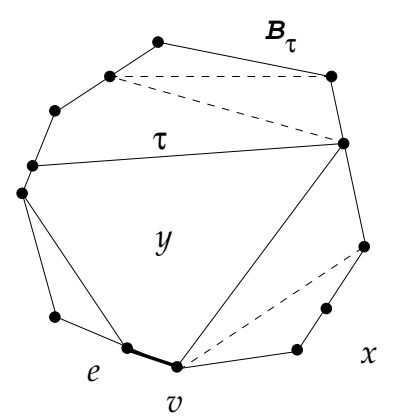

(b)

Figure 1. (a) $\tau$ is contained in a cell of $X$ and is a face of $y$. (b) $\operatorname{conv}(y)$ contains $v$ and intersects the interior of $e$.

and $\omega_{\leq x} \cap f^{-1}(y)$ is the direct product of the posets $\omega_{x}\left(\tau^{\prime}, \mathcal{B}_{\tau^{\prime}}\right)$. These posets are contractible again by induction and hence, so is their product.

We now prove Theorem 1.1 .

Proof of Theorem 1.1. Let $\omega$ denote the poset $\omega(P \stackrel{\pi}{\rightarrow} Q)$. We fix an arbitrary vertex $v$ of $Q$ and an edge $e$ which contains $v$. Let $\omega^{\prime}$ be the set of spanning, $\pi$ induced proper subconfigurations $\sigma$ of $\mathcal{A}$ such that $\operatorname{conv}(\sigma)$ contains $v$ and intersects the relative interior of $e$. We order $\omega^{\prime}$ by inclusion and define the map $f: \omega \rightarrow \omega^{\prime}$ which sends a subdivision $x \in \omega$ to the unique cell in $x \cap \omega^{\prime}$. Note that $f$ is well defined and order preserving and that $\sigma \subset \mathcal{A}$ is in $\omega^{\prime}$ if and only if the corresponding subset of vertices of $P$ intersects each one of the sets $\pi^{-1}(v), \pi^{-1}(e)-\pi^{-1}(v)$ and $P-\pi^{-1}(e)$ and spans a proper face of $P$. Hence $\omega^{\prime}$ is isomorphic to the poset of proper faces of the flag cut of $P$ at the flag of faces

$$
\emptyset \subset \pi^{-1}(v) \subset \pi^{-1}(e) \subset P
$$

(see Lemma 2.2) and is therefore homeomorphic to a $(d-3)$-sphere. One can check, as in the proof of Lemma 3.2, that the conditions of Lemma 2.1 for the map $f$ are satisfied. More specifically, let $x \in \omega, y \in \omega^{\prime}, \tau(y)$ be the set of 1-faces of $y$ whose convex hull intersects the interior of $Q$ and for each $\tau \in \tau(y)$, let $\mathcal{B}_{\tau}$ be the point configuration assigned to $\tau$ in case (ii) of Lemma 3.1 chosen so that $\mathcal{B}_{\tau}$ does not contain points of $y-\tau$ (see Figure $1 \mathrm{~b}$ ). The poset $f^{-1}(y)$ is the direct product of the posets $\omega_{X}\left(\tau, \mathcal{B}_{\tau}\right)$ which are contractible by Lemma 3.2, where $X$ denotes the trivial subdivision of $\mathcal{A}$. If $f(x)>y$, then $\omega_{\leq x} \cap f^{-1}(y)$ is the direct product of the posets $\omega_{x}\left(\tau, \mathcal{B}_{\tau}\right)$, which are again contractible by Lemma 3.2.

In the remainder of this section we assume that $P$ is a simplicial $d$-polytope, where $d \geq 3$, and that $\pi$ maps bijectively the vertex set of $P$ onto the vertex set of the polygon $Q$.

Under these assumptions, the minimal elements of $\omega(P \stackrel{\pi}{\rightarrow} Q)$ are the $\pi$-induced triangulations of $Q$. We say that two such triangulations are connected by a diagonal $\pi$-flip (see [15] for a general notion of $\pi$-flip) if they both refine a $\pi$-induced subdivision of $Q$ into polygons one of which is a quadrangle $T$ and the rest triangles, so that one triangulation is obtained from the other by "flipping the diagonal" in $T$. Let $G(Q, \pi)$ denote the graph with nodes the $\pi$-induced triangulations of $Q$ 
and edges the diagonal $\pi$-flips. Note that $G(Q, \pi)$ is the 1 -skeleton of the $(d-2)$ dimensional associahedron [18, Example 9.11] if $P$ is a simplex.

Corollary 3.3. If $P$ is a simplicial d-polytope with $d \geq 4$ and $\pi: P \rightarrow Q$ induces a bijection of the vertex sets of $P$ and $Q$, then the graph $G(Q, \pi)$ of $\pi$-induced triangulations of $Q$ and diagonal $\pi$-flips is connected.

Proof. Let $\widehat{\omega}$ denote the poset $\omega:=\omega(P \stackrel{\pi}{\rightarrow} Q)$ with a maximum element attached and $G_{\omega}$ denote the graph which is the restriction of the Hasse diagram of $\widehat{\omega}$ to its first two ranks, i.e. the set of minimal elements and those which cover a minimal element. We claim that $G_{\omega}$ is connected. Indeed, by an observation of Reiner 14, Lemma 8], it suffices to show that all strict principal order ideals $\widehat{\omega}_{<x}$ are either empty or connected or consist of exactly two incomparable elements. That $\omega$ is connected follows from Theorem 1.1. so let $x \in \omega$. If $x$ contains at most one polygon $T$ which is not a triangle, then $\omega_{<x}$ is either empty, or consists of two incomparable elements (if $T$ is a quadrangle) or is isomorphic to the poset of proper faces of an associahedron of dimension at least two, which is connected. If not, then $\omega_{<x}$ has the form $\omega_{1} \times \omega_{2}-\hat{1} \times \hat{1}$, where each poset $\omega_{i}$ has a maximum element $\hat{1}$ and cardinality at least two, and each poset of this form is easily seen to be connected.

Now observe that if $x \in \widehat{\omega}$ covers some minimal element, then this is an isolated element in $\widehat{\omega}_{<x}$. By our previous analysis, $x$ must be a proper subdivision in $\omega$, one of whose cells is a quadrangle and the rest triangles. It follows that $G_{\omega}$ is isomorphic to the first barycentric subdivision of $G(Q, \pi)$, which is therefore also connected.

Recall that a graph $G$ is $j$-connected if any induced subgraph of $G$, obtained by removing at most $j-1$ nodes, is connected and has at least two nodes. The following question is suggested by the previous corollary.

Question 3.4. Under the assumptions of Corollary 3.3 on $\pi: P \rightarrow Q$, is there a $j \geq 2$ such that the graph $G(Q, \pi)$ is always $j$-connected?

In particular, does every node of $G(Q, \pi)$ always have at least $j$ neighbors for some $j \geq 2$ ?

For similar questions and results in the context of the Baues problem see [2, 3, 9, and [14, Section 3].

\section{REFERENCES}

1. C.A. Athanasiadis, J.A. De Loera, V. Reiner and F. Santos, Fiber polytopes for the projections between cyclic polytopes, in "Combinatorics of Polytopes" (K. Fukuda and G.M. Ziegler, eds.), European J. Combin. 21 (2000), 19-47. CMP 2000:10

2. C.A. Athanasiadis, P.H. Edelman and V. Reiner, Monotone paths on polytopes, Math. Z. 235 (2000), 315-334; electronically published on July 20, 2000.

3. M. Azaola and F. Santos, The graph of triangulations of a point configuration with $d+4$ vertices is 3-connected, Discrete Comput. Geom. 23 (2000), 489-536. CMP 2000:11

4. E.K. Babson, A combinatorial flag space, Ph.D thesis, MIT, 1993.

5. H.J. Baues, Geometry of loop spaces and the cobar construction, Mem. Amer. Math. Soc. 25 (1980), 1-171. MR 81 m:55010

6. L.J. Billera and B. Sturmfels, Fiber polytopes, Ann. of Math. 135 (1992), 527-549. MR 93e:52019

7. L.J. Billera, M.M. Kapranov and B. Sturmfels, Cellular strings on polytopes, Proc. Amer. Math. Soc. 122 (1994), 549-555. MR 95a:52020 
8. A. Björner, Topological methods, in "Handbook of combinatorics" (R.L. Graham, M. Grötschel and L. Lovász, eds.), North Holland, Amsterdam, 1995, pp. 1819-1872. MR 96m:52012

9. J.A. De Loera, F. Santos and J. Urrutia, The number of geometric bistellar neighbors of a triangulation, Discrete Comput. Geom. 21 (1999), 131-142. MR 99k:52025

10. P.H. Edelman and V. Reiner, Visibility complexes and the Baues problem for triangulations in the plane, Discrete Comput. Geom. 20 (1998), 35-59. MR 99h:52013

11. D. Quillen, Homotopy properties of the poset of non-trivial p-subgroups of a group, Adv. Math. 28 (1978), 101-128. MR 80k:20049

12. J. Rambau, Polyhedral subdivisions and projections of polytopes, Ph.D. Thesis, Fachbereich Mathematik, TU-Berlin, Shaker-Verlag, Aachen, 1996.

13. J. Rambau and G.M. Ziegler, Projections of polytopes and the generalized Baues conjecture, Discrete Comput. Geom. 16 (1996), 215-237. MR 97i:52011

14. V. Reiner, The generalized Baues problem, in "New Perspectives in Algebraic Combinatorics" (L.J. Billera, A. Björner, C. Greene, R. Simion and R.P. Stanley, eds.), MSRI Book Series 38, Cambridge University Press, 1999, pp. 293-336.

15. F. Santos, On the refinements of a polyhedral subdivision, Preprint, 1999.

16. F. Santos, A point set whose space of triangulations is disconnected, J. Amer. Math. Soc. 13 (2000), 611-637. CMP 2000:12

17. B. Sturmfels and G.M. Ziegler, Extension spaces of oriented matroids, Discrete Comput. Geom. 10 (1993), 23-45. MR 94i:52015

18. G.M. Ziegler, Lectures on Polytopes, Graduate Texts in Mathematics 152, Springer-Verlag, New York, 1995. MR 96a:52011

Department of Mathematics, Royal Institute of Technology, S-100 44 Stockholm, SWEDEN

E-mail address: athana@math.kth.se 